\title{
Endothelial bedewing of the cornea in association with contact lens wear*
}

\author{
C. W. MCMONNIES AND S. G. ZANTOS \\ From the Cornea and Contact Lens Research Unit, School of Optometry, University of New South Wales, \\ Sydney, Australia
}

SUMMARY Endothelial bedewing is described as either a cluster of oedematous droplets or a constellation of leucocytes deposited on the surface of the corneal endothelium. The use of marginal retroillumination in biomicroscopy is described for the detection of endothelial bedewing. Some of the clinical characteristics of endothelial bedewing are outlined, and its occurrence in patients who are chronically intolerant to contact lens wear is reported. For these intolerant individuals endothelial bedewing may be indicative of a limited anterior uveal response associated with contact lens wear.

The early use of biomicroscopy to observe the ocular response to contact lens wear, emphasised an evaluation of superficial corneal integrity, with oedema and fluorescein staining as the chief criteria for assessment. In recent contact lens work there has been increased interest in the deeper corneal layers with the observation of vertical striae in oedematous stroma (Sarver, 1971).

Deeper still into the cornea the endothelium has received increased attention in biomicroscopy, with the observation of rapid, reversible changes in the appearance of the endothelial mosaic in association with contact lens wear (Zantos and Holden, 1977). However, the possibility of the effects of contact lens wear being manifest posterior to the endothelium has received scant attention, though it is interesting to note that a converse association has been reported. Goldberg (1970) observed that patients with posterior corneal coloured precipitates, when fitted with corneal contact lenses, suffered various forms of corneal interference and symptoms resembling overwear of contact lenses, despite only short wearing times.

The purpose of this paper is to report the observation of endothelial bedewing in some patients who show a chronic intolerance to contact lens wear. Endothelial bedewing is not a clear clinical

\footnotetext{
* Paper presented at the 3rd International Contact Lens Congress, Fiji, July 1978
}

Address for reprints: Dr S. G. Zantos, School of Optometry, University of New South Wales, PO Box 1, Kensington, New South Wales, Australia 2033 entity in relation to non-wearers of contact lenses, and its occurrence in association with contact lens wear has not previously been reported.

Stocker (1971) states that endothelial bedewing may be produced either by oedema of the endothelial cells or by precipitation of small particles of inflammatory material on the posterior surface of the cornea. In the latter case these particles include leucocytes which have been circulating in the anterior chamber (Heydt, 1923). Such cellular keratic precipitates (KP) are regarded as physiological if the cells show amoeboid movement and no tendency towards agglutination and are frequently seen in otherwise normal eyes, especially in children and adolescents (Duke-Elder, 1965). In pathological conditions such as uveitis Duke-Elder states that the deposition of KP, including leucocytes, is usually preceded by oedematous changes in the endothelium (bedewing). Thus the two forms of bedewing (oedematous and cellular) need not occur as separate clinical entities.

The classification of endothelial bedewing as either physiological or pathological may not be easy in clinical practice. However, such differentiation may be important when assessing the clinical significance of endothelial bedewing in contact lens practice, when it may be evident as a pre- or post-wear sign.

\section{Materials and methods}

By means of the slit-lamp biomicroscope with the illumination system at about 45 degrees to the observation axis, and a slit beam of 0.5 to $1.5 \mathrm{~mm}$ 


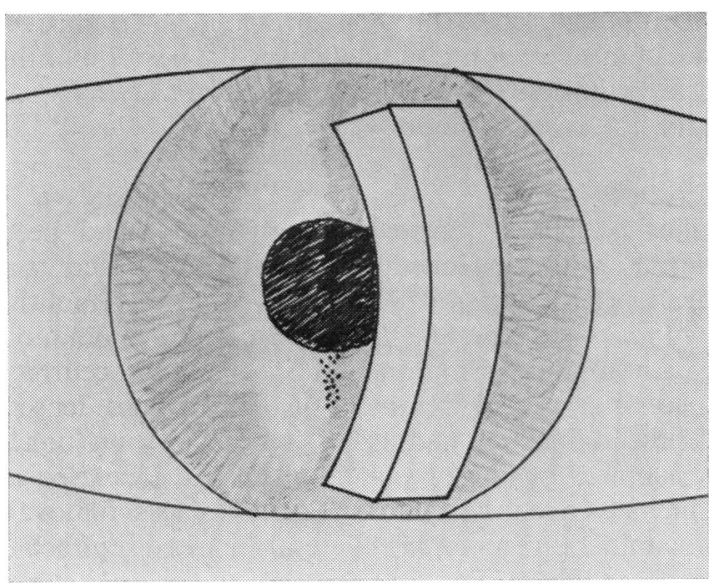

Fig. 1 Schematic representation of endothelial bedewing in the region of Ehrlich-Turk formations as seen by marginal retroillumination

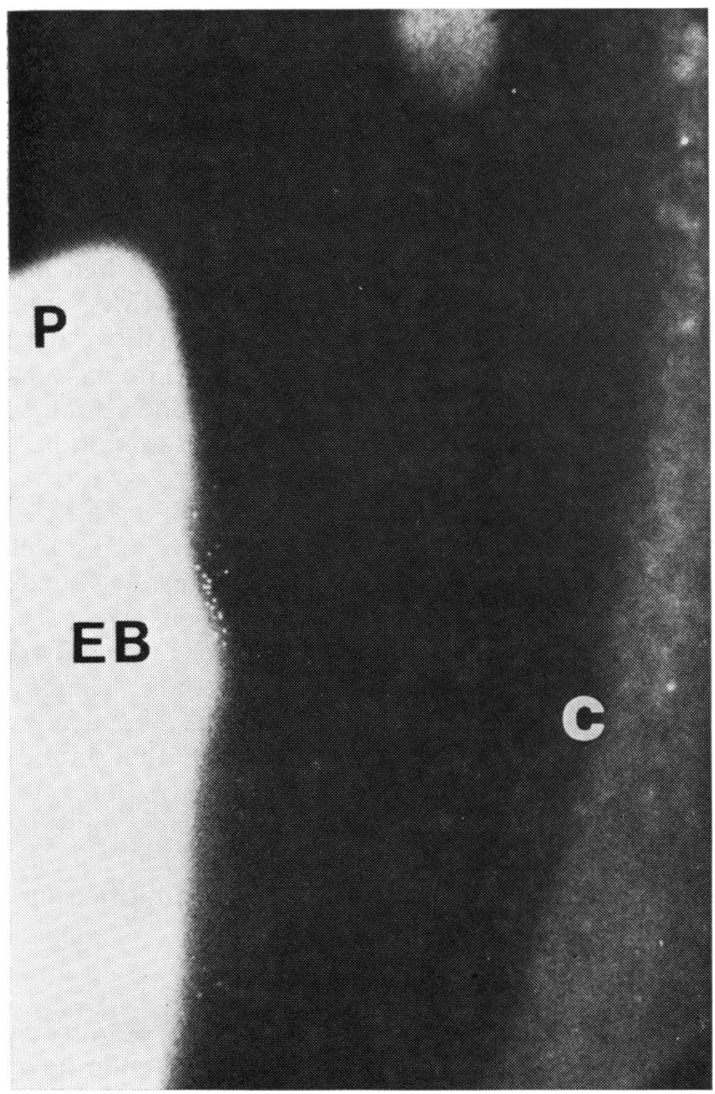

Fig. 2 Endothelial bedewing (EB) consisting of some 20 to 30 particles. The inferior margin of the pupil $(P)$ and the anterior face of the corneal parallelopiped (C) are shown. Magnification of the original slide was $\times 7 \cdot 8$

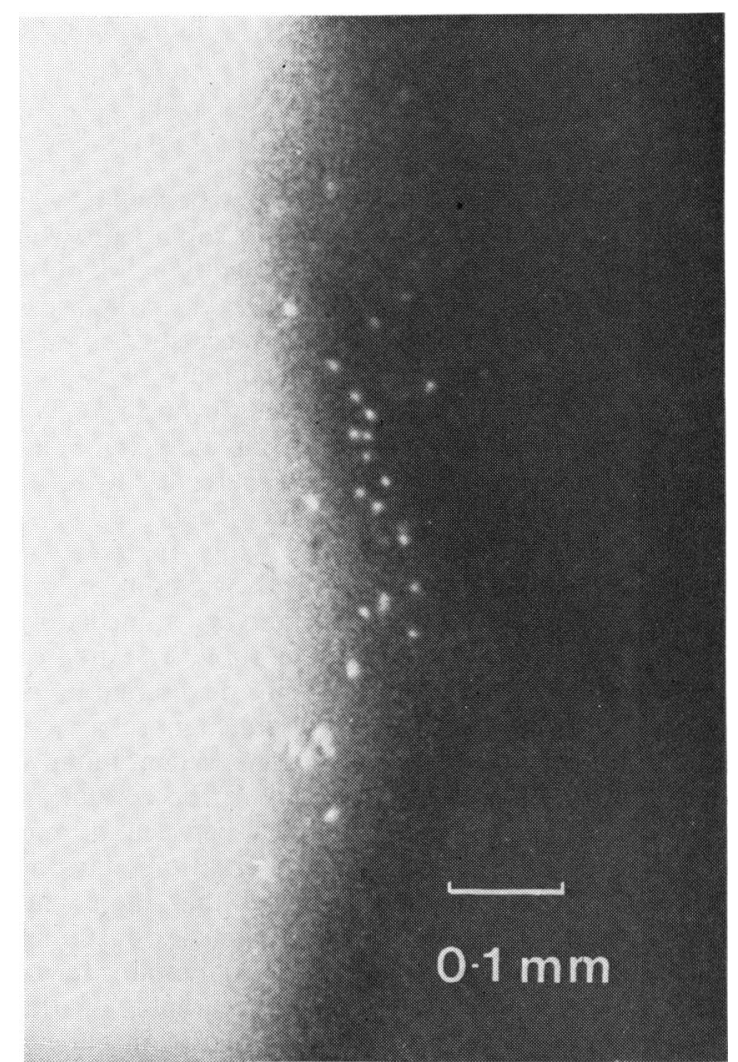

Fig. 3 Enlargement 3.4 times of Fig. 2, showing the particles of endothelial bedewing

width, the endothelial bedewing can be detected with retroillumination from the iris at magnifications of 20 times or more. The observation is carried out in the region of the cornea in line with the inferior pupil margin (the area of Ehrlich-Turk formations), with the observer's attention focused on the very edge of the beam reflected from the iris. In this critical position the droplets or white cells will be illuminated by the light reflected from the iris, and contrasted against the non-illuminated part of the iris (Figs. 1 and 2). This technique is termed marginal retroillumination (Graves, 1924), as it lies exactly between the techniques of direct and indirect retroillumination in slit-lamp biomicroscopy. The non-movement of bedewing particles contrasted with the movement of particles in the tear film following a blink is an aid in detection, but excess tear mucoid interferes with detection and observation, more so with the contact lens in situ.

In this report photographs were taken with a Nikon zoom photo slit lamp and a high magnification photographic technique described by Holden 
and Zantos (1977). Kodachrome 64 film was used for the original photographs (Fig. 2).

\section{Results}

Over a 9-month period endothelial bedewing was detected and carefully monitored in 25 patients who had either experienced marked difficulty in adapting to contact lenses or who had shown a marked loss of tolerance after a period of successful contact lens wear. These 25 patients formed a diverse group, ranging in age from 14 to 39 years (mean 27 years), with a female:male ratio of approximately $2: 1$. With the exception of one hard-lens wearer all wore or had attempted to wear soft contact lenses.

All cases of endothelial bedewing observed by the authors were detected in the region of the inferior central cornea near to or immediately below the inferior margin of the pupil. The area of bedewing varied in shape from a discrete oval cluster of particles to a less discrete, more or less dispersed Ehrlich-Turk formation. When initially detected the number of particles in the formation was found (by estimation) to vary between 20 and 50. In some cases subsequent observation: days or weeks after the first would reveal that there had been a gradual dispersion of the particles, resulting in a formation that appeared to contain fewer particles. The change in the appearance of the endothelial bedewing with time varied from patient to patient. In some cases the bedewing completely disappeared within 3 to 4 months, while in other cases it changed little over many months.

The following case reports are representative of the authors' experience.

\section{CASE 1}

This patient, an unmarried woman of 25 , began experiencing gradual loss of tolerance to her hard (PMMA) contact lenses after 5 years' successful wear. Three months after having abandoned wearing these lenses she reported for examination. Biomicroscopy revealed clear corneae and an absence of endothelial bedewing. Soft (Hema) contact lenses were supplied. These fitted well, but gave only 3 hours' tolerance after 2 weeks of attempted adaptation. Examination showed bilateral marked conjunctival injection with mild epithelial erosion in the right eye and endothelial bedewing in the left. There was no aqueous flare. After 1 week's abstinence from lens wear (during which time the lenses were altered to increase tear circulation), endothelial bedewing was now detected in both eyes, which otherwise appeared normal. Further restricted lens wear was attempted, but with limited success. A change-over from heat to chemical disinfection of the contact lenses brought no significant improvement in tolerance. Gradually over the next 2 months the bedewing resolved, but tolerance to lens wear did not exceed 5 hours.

CASE 2

This patient, a married woman of 34 , had been fitted with extended-wear hydrophilic contact lenses (Permalens), which were worn for 17 months without difficulty. She first reported for examination when marked symptoms of intolerance occurred, necessitating the removal of the contact lenses. These difficulties had been preceded by an unaccustomed fogging of vision, which had increased in intensity over the few days prior to lens removal. Examination revealed bilateral marked epithelial oedema which obscured the deeper corneal layers. Six days later the corneae were clearer, and endothelial bedewing was detected in both eyes. Over the next 2 months attempts to wear contact lenses caused sufficient symptoms to restrict usage to an occassional 2 to 3 hours' wear. The only corneal abnormality that was observed over this period was a gradual dispersion of the endothelial bedewing. Four months later minor bedewing persisted in both eyes, and contact lens wear was limited to 6 hours.

\section{CASE 3}

This patient, an unmarried woman of 39 , had had 10 years' trouble-free wear of hard (PMMA) contact lenses, when she experienced a typical overwear syndrome after the use of the lenses for 3 hours more than her usual 12 hours' wearing time. She abstained from lens wear and presented for examination 3 days later. Both eyes showed slight loss of normal corneal transparency and the presence of endothelial bedewing in cluster form in the right eye and a well dispersed constellation in the left eye. After a further 1 week's abstinence from lens wear the right eye showed a partly dispersed Ehrlich-Turk formation, and the left eye a few cells only. Attempts to wear the lenses during the next week were accompanied by an unaccustomed stinging that limited wear to 5 difficult hours. A few days later the patient reported a recurrence of overwear symptoms, though the recommended wearing period had not been exceeded. This case is still being followed up.

\section{Discussion}

The explanation for the coincidence of chronic intolerance to contact lens wear and endothelial bedewing can only be a matter of speculation at this stage. However, it is possible that, because the corneal endothelium and the anterior uvea form an intimate continuum, a disturbance to the cornea 
following contact lens wear may be accompanied by a response from the anterior uvea. The presence of inflammatory cells in the aqueous is a result of leakage through uveal blood vessels, a process which accompanies disturbances to the surrounding tissues. The subsequent deposition of cells on the corneal posterior surface is largely dependent on the thermal circulation of the aqueous and on changes in the endothelial cells predisposing to the adherence of precipitates.

According to this explanation, therefore, whenever corneal oedema occurs as a result of chronic or acute overwear of contact lenses there exists the possibility of an associated uveal response in the form of cellular escape into the aqueous. Recovery of the eye may take longer than the few days required for the disappearance of the usual clinical symptoms and signs of corneal oedema. The patients reported in this paper have shown a persistence of endothelial bedewing and concomitant reduced tolerance to contact lenses with subsequent attempts at wear. It has been a clinical experience of the authors to note intolerance to contact lenses lasting weeks or months after an episode of corneal oedema due to lens overwear. This prolonged intolerance suggests that the eyes had not completely recovered from the trauma of overwear, though normal corneal transparency had been regained. These cases have been remembered because of the difficulty in explaining to the patient the facts of good contact lens tolerance before the episode of overwear.

The apparently high incidence of endothelial bedewing in the special class of patients that suffer chronic intolerance to contact lens wear has been found to be in contrast with the incidence of endothelial bedewing in both prospective contact lens wearers and successful (non-intolerant) contact lens wearers. No cases of endothelial bedewing were found in a group of 25 prospective contact lens wearers who reported consecutively for examination, while 2 cases of bedewing have been observed in successful wearers.

The relationship between endothelial bedewing and intolerance to contact lens wear appears not to be obligatory, since the 2 conditions do not neces- sarily occur together. This is evidenced by the fact that not all intolerant lens wearers show endothelial bedewing and also by the occasional observation of endothelial bedewing in successful contact lens wearers. It would seem also that intolerance to contact lens wear and endothelial bedewing need not follow the same time course. In some cases the intolerance (though to a lesser degree) has persisted for weeks or months after the disappearance of the endothelial bedewing.

\section{Conclusion}

It is proposed that endothelial bedewing may result from an overt corneal oedema response associated with contact lens wear. The presence of endothelial bedewing may be an indication that the eye has not fully recovered from the episode of oedema, that the endothelium is still compromised, and that the prospects for successful contact lens wear are reduced. Thus it is possible that endothelial bedewing may be associated with, and an indication of, a limited uveal response to contact lens wear.

\section{References}

Duke-Elder, S. (1965). In System of Ophthalmology, Vol. VIII, Part 2, pp. 717-720. Mosby: St. Louis.

Goldberg, J. B. (1970). Biomicroscopy for Contact Lens Practice, p. 56. Professional Press: Chicago.

Graves, B. (1924). Cited by Brown, N. (1971). Visibility of transparent objects in the eye by retro-illumination. British Journal of Ophthalmology, 55, 517-524.

Heydt, R., von der (1923). The visibility of corpuscular elements within the aqueous. American Journal of Ophthalmology, 6, 822-824.

Holden, B. A., and Zantos, S. G. (1977). High magnification examination and photography with the slit lamp. In Clinical Slit Lamp Biomicroscopy, 2nd ed., pp. 348-361. Edited by R. H. Brandreth. Multimedia Communications Centre, University of California: Berkeley.

Sarver, M. D. (1971). Striate corneal lines among patients wearing hydrophilic contact lenses. American Journal of Optometry, 48, 762-763.

Stocker, F. W. (1971). The Endothelium of the Cornea and its Clinical Implications, 2nd ed., p. 84. Charles C Thomas: Springfield, Illinois.

Zantos, S. G., and Holden, B. A. (1977). Transient endothelial changes soon after wearing soft contact lenses. American Journal of Optometry, 54, 856-858. 